\title{
Imaging the Ultrasmall-Angle X-Ray Scattering Distribution with Grating Interferometry
}

\author{
P. Modregger, ${ }^{1,2, *}$ F. Scattarella, ${ }^{3,4}$ B. R. Pinzer, ${ }^{1}$ C. David, ${ }^{5}$ R. Bellotti, ${ }^{3,4}$ and M. Stampanoni ${ }^{1,6}$ \\ ${ }^{1}$ Swiss Light Source, Paul Scherrer Institut, 5232 Villigen, Switzerland \\ ${ }^{2}$ School of Biology and Medicine, University of Lausanne, 1015 Lausanne, Switzerland \\ ${ }^{3}$ Dipartimento Interateneo di Fisica "M. Merlin," Università degli Studi di Bari "Aldo Moro," 70126 Bari, Italy \\ ${ }^{4}$ Istituto Nazionale Fisica Nucleare, Sezione di Bari, 70125 Bari, Italy \\ ${ }^{5}$ Laboratory for Micro- and Nanotechnology, Paul Scherrer Institut, 5232 Villigen, Switzerland \\ ${ }^{6}$ Institute for Biomedical Engineering, UZH/ETH Zürich, 8092 Zürich, Switzerland
}

(Received 11 May 2011; published 23 January 2012)

\begin{abstract}
X-ray imaging with grating interferometry has previously been regarded as a technique providing information only in direct space. It delivers absorption, phase, and dark-field contrast, which can be viewed as parameters of the underlying but unresolved scattering distribution. Here, we present a method that provides the ultrasmall-angle $\mathrm{x}$-ray scattering distribution and, thus, allows simultaneous access to direct and reciprocal space information.
\end{abstract}

DOI: 10.1103/PhysRevLett.108.048101

Grating interferometry (GI) is a recently established phase-sensitive $\mathrm{X}$-ray imaging technique [1,2]. Commonly, the GI utilizes a phase and an absorption grating (see Fig. 1) in order to provide absorption, phase, and dark-field contrast. This is achieved by laterally scanning one of the gratings in steps that are a fraction of the gratings' pitch and acquiring an image at each step. In doing so, a so-called phase stepping curve (PSC) is obtained for each pixel, which can then be analyzed in order to yield the aforementioned contrasts [3,4]. Because of the unprecedented sensitivity [5], and in combination with an already demonstrated applicability with x-ray tubes [6], GI is believed to play an important role in future laboratorybased $\mathrm{x}$-ray imaging [7].

Usually, a flat-field PSC, $f(\phi)$, without the sample, and a sample PSC, $s(\phi)$, are acquired for each pixel. Then, a Fourier transform with respect to the phase steps is applied to the PSCs. The contrasts are obtained by calculating

$$
A=\hat{s}\left(q_{0}\right) / \hat{f}\left(q_{0}\right)
$$

for $A$, the absorption contrast, where the caret denotes the Fourier transform and $q_{0}$ is the zeroth harmonic Fourier component; by calculating

$$
P=\arg \left[\hat{s}\left(q_{n}\right)\right]-\arg \left[\hat{f}\left(q_{n}\right)\right]
$$

for $P$, the differential phase contrast (DPC), where $q_{n}$ denotes the $n$th harmonic Fourier component; and by calculating

$$
B=\left|\left[\hat{s}\left(q_{n}\right) \hat{f}\left(q_{0}\right)\right] /\left[\hat{s}\left(q_{0}\right) \hat{f}\left(q_{n}\right)\right]\right|
$$

for the dark-field contrast $B$. Assuming a Gaussian scattering distribution, the dark-field signal is related to the standard deviation of the Gaussian by $\sigma=\sqrt{-2 \ln B}$ [8]. In order to distinguish this post-detection analysis method from the one proposed in this Letter, we refer to the former as the Fourier component analysis (FCA).
PACS numbers: 87.59.-e, 07.60.Ly, 07.85.Qe, 42.30.Rx

Until now, GI has been considered to deliver information only in direct space. The "traditional" contrast modalities can be regarded as parameters of the underlying but unresolved scattering distribution. In fact, this ultrasmall-angle $\mathrm{x}$-ray scattering (USAXS) distribution constitutes reciprocal space information about the sample. In the following, we use an alternative perspective on the image formation process that allows access to the USAXS distribution with GI.

Assume a distorted wave front impinges on the detector, with $R(x)$, the scattering angle along the wave front, describing the distortion (see Fig. 1). Then, the distribution of scattering angles, $g(\alpha)$, contributing to the signal in one detector pixel is given by

$$
g(\alpha)=\int_{\text {pixel }} \delta_{D}(R(x)-\alpha) d x
$$

with $\alpha$ as the scattering angle and $\delta_{D}$ as the Dirac $\delta$ function. Upon detection, the scattering angle $\alpha$ is mapped to the lateral offset of the PSC, expressed in radians by

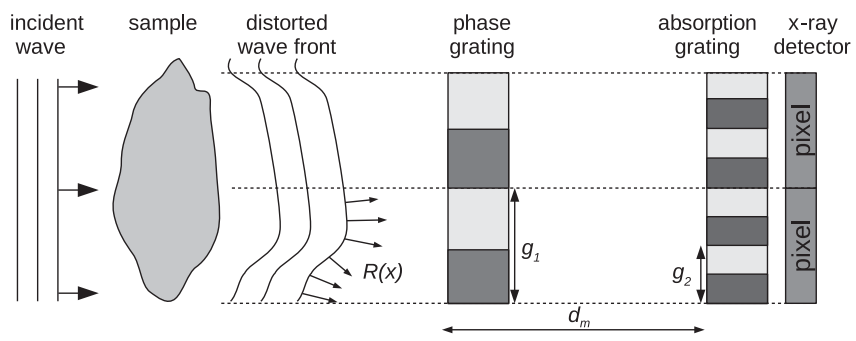

FIG. 1. Sketch of the grating interferometer. The sample is illuminated by the $\mathrm{x}$-ray beam and introduces distortions in the wave front. The distortions correspond to refraction, and $R(x)$ denotes the refraction angle as a function of the lateral spatial position $x$. The phase grating of pitch $g_{1}$ shifts the phase by $\pi$, causing a periodic interference pattern at the position of the absorption grating of pitch $g_{2}$. The interference pattern is then analyzed by laterally scanning the absorption grating. 


$$
\phi=\bmod \left(2 \pi \frac{d_{m} \alpha}{g_{2}}+\pi, 2 \pi\right)-\pi
$$

where $d_{m}$ is the intergrating distance, $m$ is the diffraction order, and $g_{2}$ is the pitch of the absorption grating [3,9]. The modulus of $2 \pi$ takes phase wrapping into account, which occurs if the scattering angle $\alpha$ corresponds to a $|\phi|>\pi$. The scattering distribution can be rewritten in terms of $\phi$ yielding $g(\phi)$. Furthermore, the PSC with the sample $s(\phi)$ equals the convolution of the PSC without the sample $f(\phi)$ (i.e., the flat PSC) with the scattering distribution $g(\phi)[8]$ :

$$
s(\phi)=f(\phi) \otimes g(\phi) .
$$

Therefore, the USAXS signal $g(\phi)$ can be accessed by deconvolving the sample PSC $s(\phi)$ with the flat $\operatorname{PSC} f(\phi)$. We denote this data analysis procedure as GI-USAXS.

The experiments were carried out at the beam line for TOmographic Microscopy and Coherent rAdiology experimenTs (TOMCAT) of the Swiss Light Source (SLS) [10] of the Paul Scherrer Institut (Villigen, Switzerland). A photon energy of $25 \mathrm{keV}$ was selected by a double multilayer monochromator, which provides an energy bandwidth of about $2 \%$. At $25 \mathrm{~m}$ distance from the source, the phase grating with a pitch of $3.98 \mu \mathrm{m}$ was located, which induced a phase shift of $\pi$. The intergrating distance of $d_{m}=121 \mathrm{~mm}(m=2)$ was close to the optimum in terms of sensitivity to density variations in the sample [11]. The phase grating as well as the absorption grating (period: $g_{2}=2 \mu \mathrm{m}$ ) were manufactured in-house by the Laboratory for Micro- and Nanotechnology [12]. The pixel size of the detector was $7.4 \mu \mathrm{m}$ with a field of view of $(12.5 \times 3.7) \mathrm{mm}$. More details about the implementation of GI at the TOMCAT beam line can be found in [13].

For the proof-of-concept experiment, we decided to use a flower as a test sample, since a strong scattering signal was expected. We acquired 20 phase steps over two periods of the absorption grating for both $f(\phi)$ and $s(\phi)$. We accumulated 100 exposures for each phase step image, to guarantee a sufficient signal-to-noise ratio during deconvolution. The exposure time was $100 \mathrm{~ms}$, and, with 4000 total images, this led to a total exposure time of approximately $7 \mathrm{~min}$. Ultimately, the number of scatter images is limited by the pitch of the absorption grating $(2 \mu \mathrm{m})$ and the resolution of the phase stepping piezo actuator $(2 \mathrm{~nm})$. The values indicate the potential for acquiring up to several hundred scatter images in future experiments.

The data analysis procedure consisted of three consecutive steps. First, the scattering distribution $g(\phi)$ was retrieved by 200 iterations of Lucy-Richardson deconvolution of the sample PSC $s(\phi)$ and the flat PSC $(\phi)$. This is known to deliver stable results even in the presence of noise [14]. Second, $g(\phi)$ values were projected into the relevant interval of $\phi \in[-\pi, \pi[$. This was necessary because there is a discrepancy between the nominal and actual period of the experimental PSCs [see Figure 2(a)]. This double period structure is caused by the manufacturing of the absorption grating. Thus, in order to ensure periodicity of the experimental PSCs, the scan was performed over two nominal periods. The resulting scattering distribution is then defined over the interval $\phi \in$ $[-2 \pi, 2 \pi[$. Using the physical constraint imposed by Eq. (5), which limits $\phi$ values to the interval $[-\pi, \pi[$, the projection of $g(\phi)$ values was justified. Third, the identity $g(-\pi)=g(\pi)$ [see Equation (5)] was used in order to symmetrize $g(\phi)$ for the subsequent moment analysis (see below).

Figure 2 shows the deconvolution procedure for two pixel positions inside and outside of the sample, which are indicated by the markers in Fig. 3(a). Figure 3 presents the USAXS signal of the entire sample for seven scattering angles (an animation showing all scatter images is available in the Supplemental Materials [15]). The global scattering distribution $g(\alpha)$ is shown in the bottom right-hand corner, which provides a reference for the numbered images. Each image in Fig. 3 represents the scattering intensity of one point in reciprocal space as a function of the spatial position. Thus, direct and reciprocal space information are simultaneously produced by GI-USAXS. Reciprocal space was sampled with an angular resolution of $\Delta \alpha=1.7 \mu \mathrm{rad}$ and a maximum scattering angle [Eq. (5)] of $\alpha_{\max }=17 \mu \mathrm{rad}$.

Previously, we stated that the traditional contrast modalities (absorption, differential phase, and dark-field) can be regarded as parameters of the underlying scattering distribution within one detector pixel. This relationship follows a simple pattern: absorption contrast corresponds to the zeroth-order moment of the scattering distribution $g(\alpha)$, the differential phase contrast to the first-order moment, and the dark-field contrast to the second centralized moment, respectively. In more detail, they can be described by the following equations:

$$
\begin{gathered}
A \rightarrow M_{0}=\int g(\phi) d \phi, \\
P \rightarrow M_{1}=\int \phi g(\phi) d \phi / M_{0},
\end{gathered}
$$

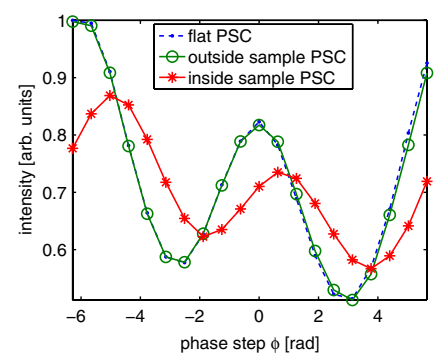

(a)

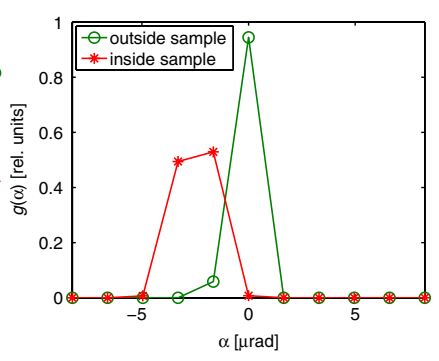

(b)
FIG. 2 (color online). Experimental demonstration of the GIUSAXS procedure. (a) One flat-field PSC $f(\phi)$ and two sample PSCs $s(\phi)$ inside and outside of the sample, as indicated in Fig. 3 (a). (b) The corresponding USAXS distributions $g(\alpha)$ in the two pixels are the result of deconvolution. 

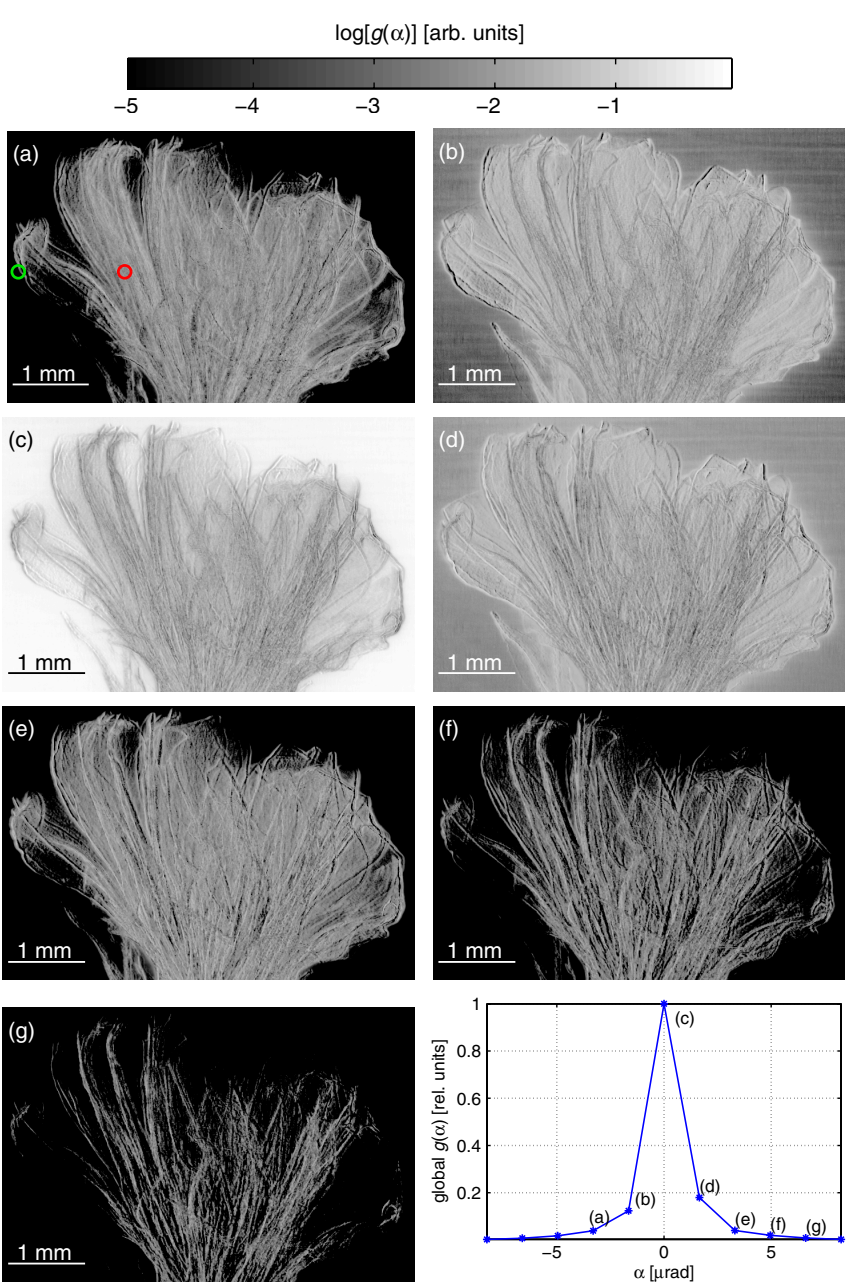

FIG. 3 (color online). USAXS signal of a flower. Seven scattering images are shown, and the bottom right-hand part of the figure indicates the position of the individual images on the global scattering curve.

$$
\begin{gathered}
\sqrt{-2 \ln B} \rightarrow \sigma=\sqrt{\int\left(\phi-M_{1}\right)^{2} g(\phi) d \phi / M_{0}}, \\
s=\int\left(\phi-M_{1}\right)^{3} g(\phi) d \phi /\left(M_{0} \sigma^{3}\right),
\end{gathered}
$$

with the skewness as $s$, which quantifies the asymmetry of the scattering distribution [16]. In this sense, the traditional contrast modalities are included in the USAXS distributions and GI-USAXS can be regarded as a generalization of FCA. In turn, the results of FCA can be used to validate the retrieved scattering distributions.

Figure 4 compares the traditional contrasts to their corresponding moments. The difference images are provided in the Supplemental Materials [15]. The excellent agreement of absorption and zeroth moment (mean relative difference over the field of view: $0.3 \%$ ) and the good agreement of both scattering widths $\sigma$ (mean relative difference over the field of view: $12 \%$ ) demonstrate the

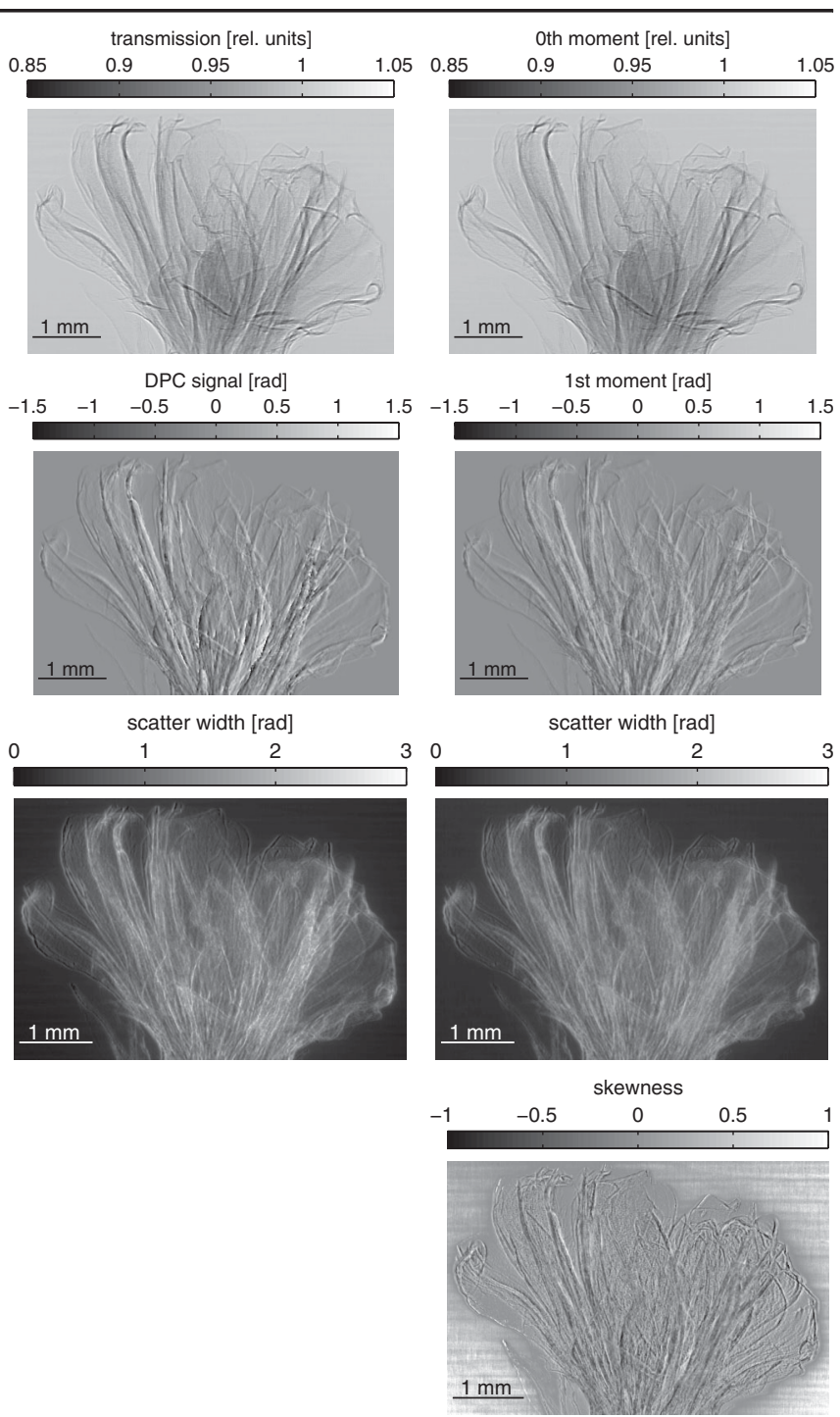

FIG. 4. Comparison between FCA and the moments of the pixelwise USAXS distributions. On the left-hand side, the absorption, differential phase, and dark-field contrast (from top to bottom) are shown. On the right-hand side, the corresponding moments of the USAXS distribution are shown. The contrast corresponding to the skewness is not accessible by FCA.

validity of GI-USAXS. However, a small discrepancy between the DPC and the first moment signal was found. This can be explained by the fact that the standard FCA method implicitly assumes a symmetric scattering distribution. Thus, the observed asymmetry of the scattering distributions leads to systematic errors in the retrieval of the DPC values by FCA. This issue is resolved by GI-USAXS, which provides additionally access to the previously unobtainable skewness of the scattering distribution. Further, it has been indirectly shown that the dark-field signal originates from scattering variations within one detector pixel by using a crystal-based USAXS setup [17]. We have additionally directly verified this claim with a GI setup and identified the second moment of the scattering distribution with the dark-field signal. 

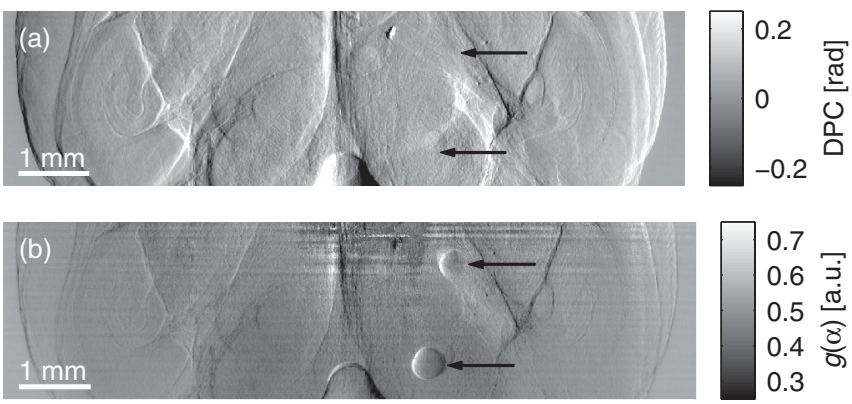

FIG. 5. Comparison between standard analysis and the GIUSAXS method at the example of a paraffin-embedded rat brain. (a) DPC image and (b) USAXS signal at the slope of the global scattering signal (i.e., $\alpha=1.7 \mu \mathrm{rad}$ ). The superiority in terms of sensitivity of GI-USAXS to FCA is clearly exemplified by the visibility of the bubbles.

Finally, we demonstrate an indication of improved sensitivity of the GI-USAXS method compared to FCA with an argumentative sound, albeit unusual, example. The sample for this experiment was a paraffin-embedded rat brain immersed in liquid paraffin. During data acquisition, a bubble was present only in some images. Normally, the bubble would be considered an artifact, appearing as such in the DPC image [Fig. 5(a)]. However, while the bubble is not visible in the DPC image, it is clearly visible in the scattering image [Fig. 5(b)]. Since the same data (i.e., accumulated images) were used for both analysis procedures, the signal-to-noise ratio of the raw images was identical. Thus, we can conclude that the GI-USAXS indeed provides a higher sensitivity compared to FCA. The horizontal stripes in Fig. 5(b) constitute artifacts that are due to vibrations of the monochromator. The artifacts may be removed by applying appropriate post-detection algorithms (e.g., wavelet filtering [18]).

Small-angle x-ray scattering (SAXS) provides direct access to reciprocal space by focusing the beam on the sample and acquiring the scattered intensity by an area detector positioned several meters downstream. The angular resolution of reciprocal space mapping is limited by the sample-detector distance and the pixel size of the detector. For the following comparison, the cSAXS beam line [19] at the SLS was used as a representation of a state-of-the-art setup. Here, a pixel size of $172 \mu \mathrm{m}$ and a sample-detector distance of $7150 \mathrm{~mm}$ implied an angular resolution of $\Delta \alpha \approx 24 \mu \mathrm{rad}$, which is 1 order of magnitude larger than the angular resolution of GI-USAXS $(\Delta \alpha \leq$ $2 \mu \mathrm{rad})$. This can be regarded as a first indication that SAXS and GI-USAXS deliver access to reciprocal space on complementary angular scales.

In conclusion, we established GI-USAXS as a promising post-detection analysis method for GI. The successful experimental demonstration validates the underlying theoretical framework, and, thus, GI-USAXS is expected to have a positive impact on future investigations about the fundamental physics of x-ray scattering. In particular, it was already possible to relate the dark-field contrast to the second moment of the scattering distribution.

In comparison to the traditional analysis method, we extended the number of complementary contrasts from three to potentially hundreds. Further, GI-USAXS is directly applicable to laboratory-based GI setups. Most importantly, GI-USAXS simultaneously allows access to direct and reciprocal space information about the sample. Therefore, GI-USAXS offers the potential for a widespread application in the fields of materials science and biomedical imaging.

We would like to acknowledge Simon Rutishauser (Laboratory for Micro- and Nanotechnology, Paul Scherrer Institut, Switzerland) for the manufacturing of the gratings. This study was supported by Centre d'Imagerie BioMédicale (CIBM) of the UNIL, UNIGE, HUG, CHUV, and EPFL and the Leenaards and Jeantet Foundations.

*peter.modregger@psi.ch

[1] C. David, B. Nöhammer, H. H. Solak, and E. Ziegler, Appl. Phys. Lett. 81, 3287 (2002).

[2] A Momose et al., Jpn. J. Appl. Phys. 42, L866 (2003).

[3] T. Weitkamp et al., Opt. Express 13, 6296 (2005).

[4] F. Pfeiffer et al., Nature Mater. 7, 134 (2008).

[5] F Pfeiffer et al., Phys. Med. Biol. 52, 6923 (2007).

[6] F. Pfeiffer, T. Weitkamp, O. Bunk, and C. David, Nature Phys. 2, 258 (2006).

[7] M. Bech et al., Z. Med. Phys. 20, 7 (2010).

[8] Z.-T. Wang, K.-J. Kang, Z.-F. Huang, and Z.-Q. Chen, Appl. Phys. Lett. 95, 094105 (2009).

[9] T. J. Suleski, Appl. Opt. 36, 4686 (1997).

[10] M. Stampanoni et al., in Synchrotron Radiation Instrumentation, edited by J.-Y. Choi and S. Rah, AIP Conf. Proc. No. 879 (AIP, New York, 2007), p. 848.

[11] P. Modregger et al., Opt. Express 19, 18324 (2011).

[12] C. David et al., Microelectron. Eng. 84, 1172 (2007).

[13] S. A. McDonald et al., J. Synchrotron Radiat. 16, 562 (2009).

[14] M. Kumar Singh, U. Shanker Tiwary, and Y.-H. Kim, Eurasip J. Adv. Sig. Pr. 2008, 365021 (2008).

[15] See Supplemental Material at http://link.aps.org/ supplemental/10.1103/PhysRevLett.108.048101 for an animation showing all scatter images of the flower and for the difference images of the absorption, differential phase, and dark-field contrasts and their corresponding moments of the scattering distribution.

[16] F. James, Statistical Methods in Experimental Physics (World Scientific, Singapore, 2006), 2nd ed.

[17] M. Yashiro et al., Opt. Express 18, 16890 (2010).

[18] B. Münch, P. Trtik, F. Marone, and M. Stampanoni, Opt. Express 17, 8567 (2009).

[19] O. Bunk et al., New J. Phys. 11, 123016 (2009). 